\title{
Fault-Tolerance Experiments with a Kinematically Redundant Holonomic Mobile Robot
}

\author{
O. N. Şahin ${ }^{1}$, O. Çelik ${ }^{1}$ and M. İ. C. Dede ${ }^{1}$ \\ ${ }^{1}$ Izmir Institute of Technology, Izmir, Turkey, e-mail: osmansahin, onurcelik, \\ candede@iyte.edu.tr
}

\begin{abstract}
Indoor locomotion with mobile robots has found applications in industrial part moving, factory floor investigation and cleaning tasks. Holonomic mobile robots have the advantage of moving in tight and winding passages without the need for steering. In this study, a four omnidirectional wheeled mobile robot is considered. The mobile robot is kinematically redundant having four of these wheels and this is used in providing fault-tolerance due to a malfunction in one of the wheels. In this paper, the redundancy resolution for this mobile robot is explained providing a solution to a fault in one of the wheels during operation. A top level controller to compensate for the orientation errors is introduced. Finally, experimental set-up is presented along with the result of the fault-tolerance experiments.
\end{abstract}

Key words: Holonomic Mobile Robot, Fault Tolerance, Kinematic Redundancy, Omnidirectional Wheels.

\section{Introduction}

Mobile robots find application domains in an increasing trend. They were first employed autonomous ground vehicles (AGV) in factory floors [1] and later mobile robots entered the households as automatic cleaning agents [2]. Among the key components of these robots, localization equipment, battery management and locomotion mechanism are perhaps the most important ones. In terms of locomotion, mobile robots can be classified as wheeled, tracked and legged mobile robots.

A subtype of indoor wheeled mobile robots is the holonomic mobile robots. Holonomic refers to the relationship between controllable and total degrees of freedom of a robot. If the controllable degree of freedom is equal to total degrees of freedom, the robot is holonomic. A robot which has omni-directional wheels is a good example of holonomic mobile robots. Holonomic mobile robots are capable of translational motion in two degrees-of-freedom (DoF) on a plane and a single DoF rotational motion about the normal to the plane independently and simultaneously. As a result of this advantage, holonomic mobile robots are widely used 
in many applications, especially in tasks carried out in narrow spaces such as transporting of goods in hospitals, public cleaning, factories and sheltered workshops for disabled people [3].

One problem that arises with the use of autonomous vehicles is the need of fault tolerance against the possible malfunction of component during operation or manufacturing faults. In this study, mechanism level fault tolerance is worked out by introducing redundancy to a holonomic mobile robot. The faults considered in this paper are a single wheel malfunction and faults during manufacturing and assembly of the mobile robot. The next section provides a brief overview of holonomic mobile robots and the omni-directional wheels used for these robots. In the following section, the holonomic robot designed and built in this work is presented along with the kinematics equations. The redundancy resolution algorithm that enables the fault tolerance due to a malfunction of a wheel is explained and the top level controller for compensating the orientation errors, which occur due to the malfunctioning wheel and/or manufacturing errors, is introduced. Finally, experimental results are provided and discussed.

\section{Overview of Holonomic Mobile Robots}

Holonomic motion of ground vehicles can be constructed with ordinary caster wheels [4] or special omnidirectional wheels [5] (mecanum and universal). Holonomic robots, which have caster wheels, firstly changes wheel orientation according to motion direction and then robot moves. This procedure brings delay in operation and robot cannot perform a smooth continuous motion. Omnidirectional wheels allow motion that is perpendicular to the rotating direction of the wheel through smaller free rollers which are placed on the outer diameter of the wheel. In holonomic mobile robots that use caster type of wheels, at least three caster wheels and six actuators among which 3 actuators are required for rotation of wheels and 3 actuators are required for changing the wheels' orientation. In holonomic mobile robots that use omnidirectional wheels, only three universal or four mecanum wheels and an actuator for every wheels are enough. Therefore, omnidirectional wheels are more efficient for holonomic ground robots in terms of minimized use of the actuators. Universal wheels (Figure 1) are selected for the locomotion of developed holonomic mobile robot in this study. 


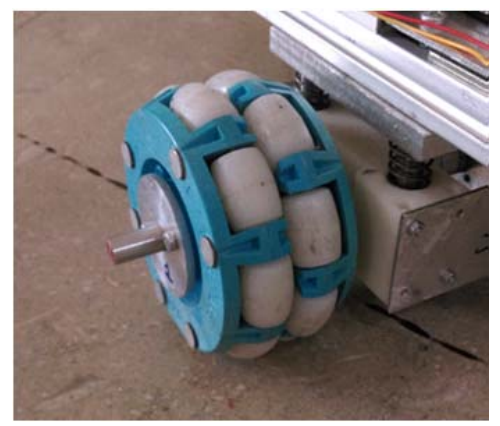

Fig. 1 The omni-wheel used in this study.

Omnidirectional wheels have some problems despite their advantages. Most important disadvantage of them, which affects motion capability of holonomic mobile robot, is that outer circle of wheel is completed with radius of each roller and this is not a smooth circle. Although effect of this problem is decreased with two row rollers, the problem causes vibration on mobile robot, decreases roadholding and makes it difficult to carry out odometry from encoders mounted on wheel actuators. While rollers are placed perpendicular to the circular plane of the wheels in universal wheels, rollers are placed with an angle in mecanum wheels. This enhances the road-holding of the robot so that mecanum wheels are commonly preferred for industrial holonomic lifting and carrying robots [6].

\section{Design and Governing Equations of the Holonomic Mobile Robot}

In this study, a mobile robot is designed to have kinematic redundancy by using 4 universal wheels. It is actually enough to use 3 universal wheels for constructing a holonomic mobile robot. Mobile robots can be employed at distant or dangerous environments for human, such as in space or in nuclear reactors. Tolerating the faults emerged during operation is crucial for operational success. Therefore, main reason of selecting this configuration is that if any wheel is broken down while in operation, robot can tolerate this fault and continue the operation with three wheel without losing its holonomic property.

Figure 2 represents location of mobile robot according to global coordinate frame and wheel velocities of the robot. Where $V_{i}$ and $\omega_{i}(i=1,2,3,4)$ are linear and angular velocities of wheels respectively, $\theta$ is orientation of mobile robot. All four wheels are placed so that the angles between the neighboring wheels are $90 \mathrm{de}-$ grees. Distances between wheels and center of mass are same $(250 \mathrm{~mm})$ and shown with the parameter L in Figure 2. 


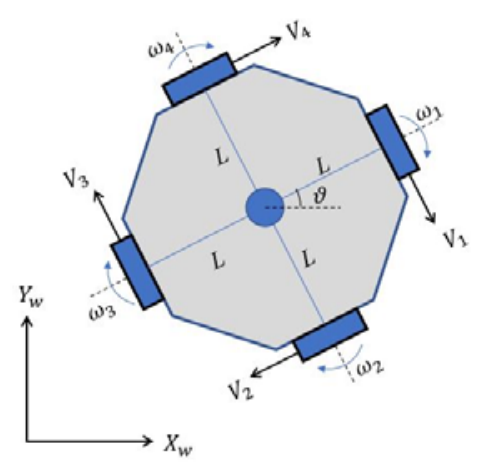

Fig. 2. Top view of four wheeled mobile robot

Kinematic equations of mobile robot derived by assuming that there is no slippage during operation are given below,

$$
\begin{gathered}
V_{x}=V_{1} \cdot \sin \theta-V_{3} \cdot \sin \theta-V_{2} \cdot \cos \theta+V_{4} \cdot \cos \theta \\
V_{y}=-V_{1} \cdot \cos \theta+V_{3} \cdot \cos \theta-V_{2} \cdot \sin \theta+V_{4} \cdot \sin \theta \\
\omega_{v}=-\left(V_{1}+V_{2}+V_{3}+V_{4}\right) / L \\
{\left[\begin{array}{c}
V_{x} \\
V_{y} \\
\omega_{v} \cdot L
\end{array}\right]=J \cdot\left[\begin{array}{l}
V_{1} \\
V_{2} \\
V_{3} \\
V_{4}
\end{array}\right]} \\
J=\left[\begin{array}{cccc}
\sin \theta & -\cos \theta & -\sin \theta & \cos \theta \\
-\cos \theta & -\sin \theta & \cos \theta & \sin \theta \\
1 & 1 & 1 & 1
\end{array}\right]
\end{gathered}
$$

Because of the redundancy, Jacobian matrix given in Equation 5 is not a square matrix. Hence, pseudo inverse method used to find minimum norm of wheel velocities. Inverse pseudo Jacobian matrix, $\mathrm{J}^{+}$, for robot is derived as below:

$$
\begin{gathered}
{\left[\begin{array}{l}
V_{1} \\
V_{2} \\
V_{3} \\
V_{4}
\end{array}\right]=J^{+}\left[\begin{array}{c}
V_{x} \\
V_{y} \\
-\omega_{v} \cdot L
\end{array}\right]} \\
J^{+}=J^{T}\left(J \cdot J^{T}\right)^{-1}
\end{gathered}
$$




\section{Fault Tolerance Aspects}

Fault tolerance studies in literature are achieved in two aspects; fault diagnosis and fault tolerance. Also, faults can be occurred in software or hardware of a mobile robots. This study is focused on tolerating hardware faults of developed in a holonomic mobile robot.

One of fault tolerance scenario is the case of failure in the actuators coupled to the wheels. The method of weighted pseudo inverse of Jacobian matrix $\left(\hat{J}_{w}^{+}\right)$is applied to deal with this type of fault. The direct kinematics equation is modified to Equation 8 and inverse kinematics making use of $\hat{J}_{w}^{+}$is calculated as shown in Equation 9.

$$
\begin{gathered}
{\left[\begin{array}{l}
V_{1} \\
V_{2} \\
V_{3} \\
V_{4}
\end{array}\right]=\hat{J}_{w}^{+}\left[\begin{array}{c}
V_{x} \\
V_{y} \\
-\omega_{v} L
\end{array}\right]} \\
\hat{J}_{w}^{+}=\widehat{W}^{-1} \cdot \hat{J}^{T} \cdot\left(\hat{J} \cdot \widehat{W}^{-1} \cdot \hat{J}^{T}\right)^{-1}
\end{gathered}
$$

$W$ is a diagonal weighing matrix.

$$
\widehat{W}=\left[\begin{array}{cccc}
W_{1} & 0 & 0 & 0 \\
0 & W_{2} & 0 & 0 \\
0 & 0 & W_{3} & 0 \\
0 & 0 & 0 & W_{4}
\end{array}\right]
$$

$W_{1}, W_{2}, W_{3}, W_{4}$ each represents the chosen weighing constant for the designated motor. Increasing any $W_{i}$ constant with respect to other three, in Equation 7, will decrease the corresponding motor's contribution in operation. If a weight is to be chosen infinite, the desired motion will be carried out with the other three since the system is already redundant. The calculation of the linear velocity requirement of one of wheels for a desired task space velocity profile is given in Equation 11.

$$
\begin{aligned}
V_{1}= & -\left[0.5 /\left(W_{1}+W_{2}+W_{3}+W_{4}\right)\right]\left[\omega_{V} \cdot L\left(W_{2}+W_{4}\right)\right. \\
& +V_{Y}\left(\operatorname{Sin} \theta\left(W_{2}-W_{4}\right)+\operatorname{Cos} \theta\left(W_{2}+2 W_{3}+W_{4}\right)\right) \\
& \left.+V_{x}\left(\operatorname{Cos} \theta\left(W_{2}-W_{4}\right)-\operatorname{Sin} \theta\left(W_{2}+2 W_{3}+W_{4}\right)\right)\right]
\end{aligned}
$$

Fault tolerance is adapted to the redundant mobile robot system to be used in case of losing power in one motor completely or decrement in motor efficiency. In order to evaluate the efficiency of this algorithm, the failure is generated artificially in the control interface by cancelling motor control or decreasing its control input signal. 
Additionally, two different sets of omnidirectional wheels with different friction properties are used for further fault tolerance tests. The rollers of two universal wheels are synthetic rubber coated polypropylene and other two are nylon. This fault causes various road-holding performance of the robot in each direction.

The other faults focused on in this study are manufacturing errors and deficiency of omnidirectional wheels. Even if angles between wheels are set as 90 degrees, because of manufacturing process, these angles can have small deviation, which can result in uneven input from the wheels. Also, because of disadvantages of omnidirectional wheels mentioned in Section 2, road-holding of any wheel can be different from others. These problems cause undesirable orientation changes. In order to deal with this problem and to control the angular velocity of mobile robot, a top level closed-loop control is developed at velocity level that uses the angular velocity of robot measured through a gyroscope attached on mobile robot as the feedback signal. Figure 3 shows the Top-Level controller algorithm.

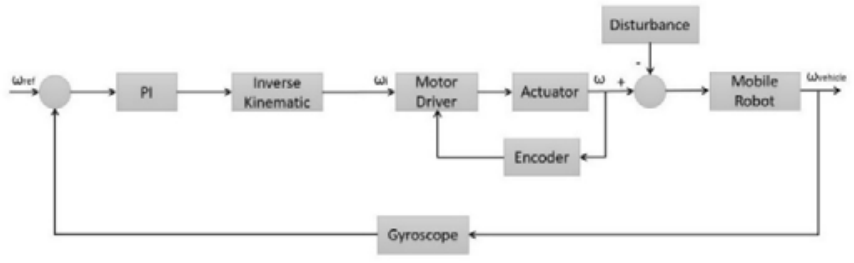

Fig. 3. Top Level Control Algorithm

In Figure 3, reference input $\omega_{\text {ref }}$ is the desired angular velocity of the mobile robot. After the required velocity change is regulated by the PI controller, the task level velocities are decomposed to wheel velocities and low-level wheel actuator controllers regulate the wheel speeds accordingly. The Top-Level controller (PID or PI) parameters are selected experimentally in order to make the controlled system to have a $5 \mathrm{rad} / \mathrm{sec}$ bandwidth. Red dashed lines show the $3 \mathrm{~dB}$ range in Figure 4. PID controller (blue line) has a resonant frequency at about $3 \mathrm{rad} / \mathrm{sec}$. However, with PI controller (orange line), it was possible to have a flat magnitude plot until $5 \mathrm{rad} / \mathrm{sec}$. The parameters of PI controller are $\mathrm{K}_{\mathrm{p}}=4, \mathrm{~K}_{\mathrm{i}}=1,25$.

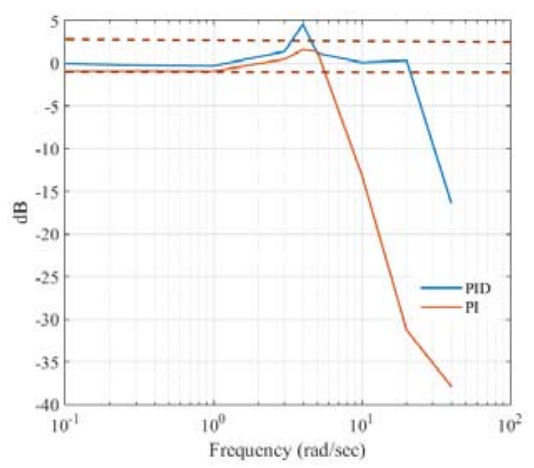

Fig. 4. Bode plot for PID and PI controller 
System with the Top-Level controller produced the transient state characteristics listed in Table 1.

Table 1. Top-Level Controller Transient Response Characteristics.

\begin{tabular}{ll}
\hline Parameters & \\
\hline $\mathrm{t}_{\mathrm{r}}$ & $0.614 \mathrm{sec}$ \\
$\mathrm{t}_{\mathrm{p}}$ & $0.796 \mathrm{sec}$ \\
$\mathrm{t}_{\mathrm{s}}($ for 2\%) & $8 \mathrm{sec}$ \\
Percent Overshoot & 18 \\
\hline
\end{tabular}

\section{Experimental Verification of Fault Tolerance Features}

The robot manufactured in this study (Figure 5) is a four-wheeled redundant holonomic mobile robot. The robot is driven with four 24V DC motor (Dunkermotoren G30.0) and Maxon ADS 50/10 motor driverw. Energy need is supplied with two $12 \mathrm{~V}$ lead acid batteries. Energy supply unit has a step-up circuitry to increase voltage from $12 \mathrm{~V}$ to $24 \mathrm{~V}$ for driving actuators and step-down circuitry to supply energy to sensors and the DAQ system. UEISIM 600-1G GigE Simulink target Cube which have two modules (analog input and output modules) is utilized for data acquisition. Data transfer between host pc to target UEISIM 600-1G is provided by UDP protocol. The wireless communication is used to embed control algorithms, which are generated with Matlab Simulink, into the robot's controller and for monitoring and logging the data from UEISIM DAQ during experimental studies. Wheel angular velocities are measured with magnetic encoders attached at the rear end of the motors. Also developed mobile robot consists of an angular velocity measurement unit (analog gyroscope) and eight infrared sensor (SHARP GP2Y0A02YK0F) to detect obstacles surrounding of the robot.

The controller embedded in the mobile robot utilizes the weighted pseudo inverse of the Jacobian matrix as explained in Section 4. In case of the existence of a faulty actuator, all actuator motion demands are updated by changing weighing constant of each wheel in weighing matrix. During the experiments, faults are generated manually in the algorithm as a performance drop in one of the actuators. The objective of this study does not include fault detection therefore, it is assumed that fault is detected and the time of occurrence is known.

The fault is defined as proportional to the performance loss. During the experiments one actuator is subjected to performance loss up to $\% 70$. According to the test scenario, while the mobile robot is following a linear path, one of its actuators providing this linear motion is subjected to a performance loss at $5^{\text {th }}$ second. 


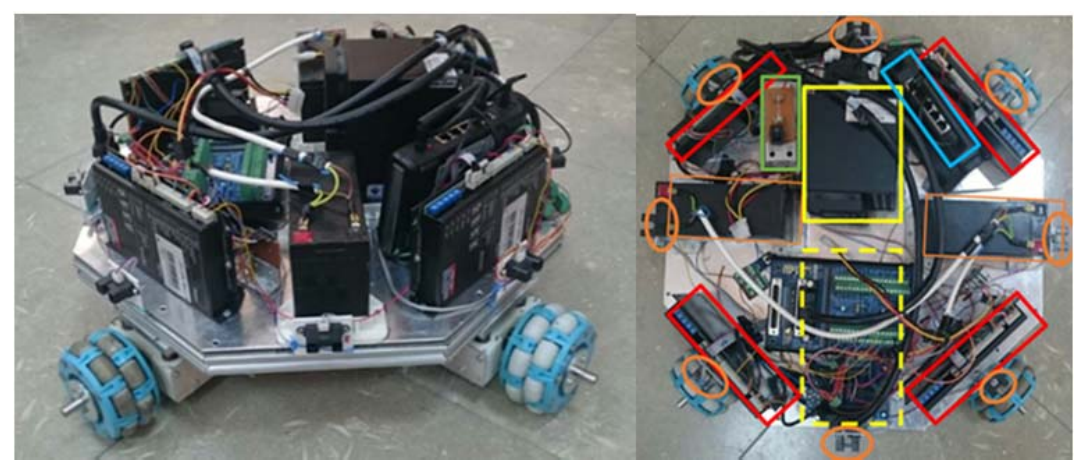

Fig. 5. Four omnidirectional-wheeled holonomic mobile robot (left: Perspective view, right: top view ; in the top view yellow box is the UEISIM DAQ, blue box is the wireless router, red boxes are the motor drivers, orange boxes are the batteries, orange ellipses are the infrared sensors, green box is the power inverter and dashed yellow box is the $\mathrm{I} / \mathrm{O}$ cards.)

The non-idealities such as, skewness in actuator axes, friction coefficient difference of the wheels and translation in mass center, leads to the need of observing fault tolerance algorithm in different motion directions selected as $\mathrm{x}$ - and $\mathrm{y}$ directions. The experiments are run for different levels of performance loss and the performance loss of the actuators is increased by 10 percent until the actuators are completely disabled. The angular velocities of wheels measured with magnetic encoders are illustrated for the motion along x-direction in Figure 6 (a) and ydirection in Figure 6 (b). In both experiments, motion of the robot is initiated at second 2 and the performance loss for one actuator is issued at second 5 . The performance loss level that is kept at $50 \%$.

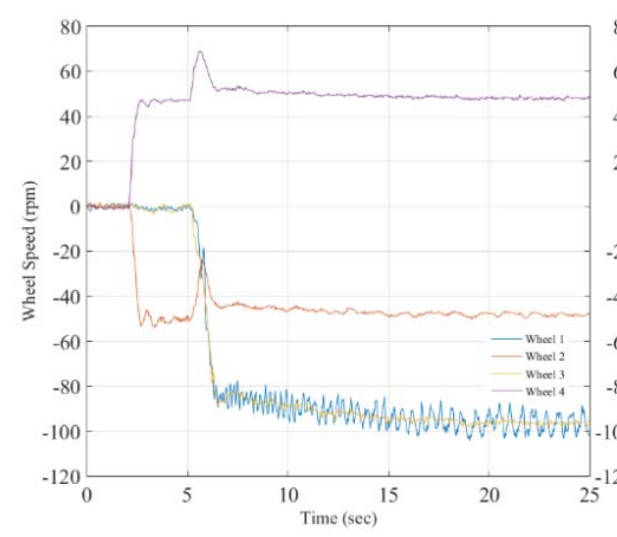

(a)

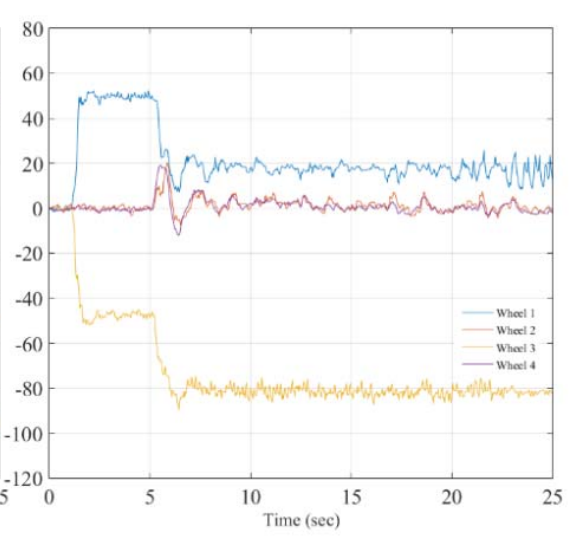

(b)

Fig. 6. Velocity distribution of mobile robot for experiment (a)- moving in $x$ direction, (b)- moving in y direction 
Wheels 1 and 3 that are responsible for the motion along y-direction have the rollers with relatively smaller friction coefficients. As a consequence of this, it can be observed from Figure 6 (a) that these two wheels start to operate at fast speeds in order to compensate for the faulty wheel 2 . The encoder reading from the faulty wheel 2 shows that the performance did not drop to $50 \%$ however, this angular velocity measurement is due to the rolling of the wheel with friction as the mobile robot moves along the $\mathrm{x}$-direction.

In Figure $6(\mathrm{~b})$, wheels 1 and 3 provide the motion along the y-direction. When $50 \%$ fault is issued to wheel 1, its angular velocity drops to $50 \%$. Wheels 2 and 4 with relatively better friction coefficients hold the road better and thus provide the necessary traction with minimal effort. Therefore, the angular velocity contributions of wheel 2 and 4 are very limited as it can be observed from Figure 6 (b).

It can also be observed from Figure 7 (a) and (b) that after the transient state the angular velocity of the mobile robot is kept at about zero condition in both of the experiments. This shows that the Top-Level controller is successful in regulating the angular velocity of the mobile robot independent of the difference in roadholding characteristics of the wheels.

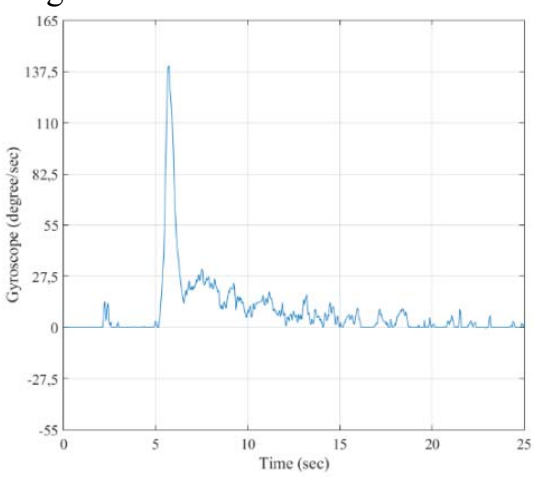

(a)

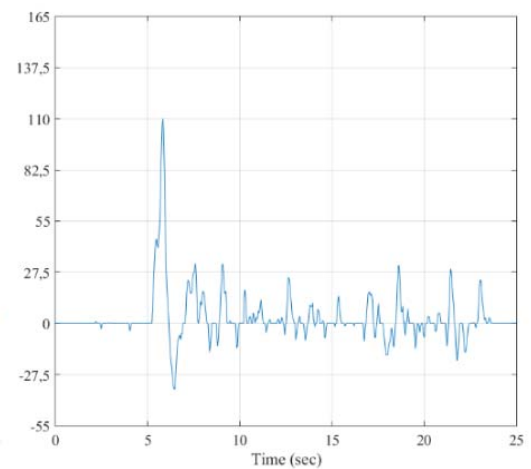

(b)

Fig. 7. Gyroscope measurements of mobile robot with Top-level controller (a)- moving along $x$ direction, (b)- moving along y direction

\section{Conclusion}

In this study, experimental results of fault tolerance algorithm devised for a four omnidirectional wheeled holonomic mobile robot are presented. Faults focused on this study are the performance loss of any wheel of robot and undesired orientation changes caused by manufacturing errors and uneven friction coefficients of the omnidirectional wheel. Because the mobile robot is designed to have kinematic redundancy, performance loss or disability of any wheel during robot in task can be tolerated with other the three wheels and the robot can perform the 
task without losing its holonomic motion ability. This fault is tolerated by using weighted pseudo inverse of the Jacobian matrix derived for the robot's kinematics.

The fault tolerance method developed in this work is applied in robot controller and fault tolerance tests are carried out. According to test results given in previous section, fault tolerance algorithm tolerates performance loss of any wheel during a motion in any direction. However, the test results for both direction are different from each other because of the differences in wheels' friction characteristics (rollers of two wheels are coated synthetic rubber, other two are nylon).

It should be noted that during fault tolerance tests performed without top-level controller, mobile robot rotates uncontrollably since the wheels, which compensate angular velocity changes of the robot, have different road-holding capabilities. Top-level controller is necessary to eliminate this fault in the system due to uneven wheel characteristics.

\section{References}

1. Takashi G., Koh-ichi I. and Hirokazu M.: The Development of a Fully Autonomous Ground Vehicle (FAGV). In: Intelligent Vehicle '94 Symposium, IEEE, pp. $62-67$ (1994)

2. Joseph L. J.: Robots at the Tipping Point. In: IEEE Robotics \& Automation Magazine, pp.76$78(2006)$

3. John. M. E.: HelpMate: an autonomous mobile robot courier for hospitals. In: Intelligent Robots and Systems '94, pp.1695-1700 (1994)

4. Robert H. and Oussama K.: Development and Control of a Holonomic Mobile Robot for Mobile Manipulation Tasks. In: The International Journal of Robotics Research. pp. 1066-1074 (2000)

5. Martin U., Karl I.: Analysis, Design, and Control of an Omnidirectional Mobile Robot in Rough Terrain. In: Journal of Mechanical Design. (2009)

6. Olaf D., Aparna B., Glen B., Johan P. and Sylvester T.: Improved Mecanum Wheel Design for Omni-directional Robots. In: Australasian Conference on Robotics and Automation. pp 117$121(2002)$ 